\title{
RENDIMIENTO COGNITIVO Y SÍNTOMAS AFECTIVOS EN LA FASE AGUDA Y TRES MESES DESPUÉS DE UN ICTUS ISQUÉMICO
}

\author{
Luis GutiÉrReZ-CABEllo ${ }^{1}$, ÁNgel Aguilar-Alonso ${ }^{1}$, ANTONio DÁvalos ${ }^{2}$ Y \\ SALVADOR PEDRAZA ${ }^{2}$ \\ ${ }^{1}$ Facultad de Psicología, Universidad de Barcelona \\ ${ }^{2}$ Instituto de Diagnóstico por Imagen (IDI), Hospital Universitario de Gerona Dr. Josep Trueta
}

\begin{abstract}
Resumen: Después de un ictus, se ha descrito deterioro de la cognición, del humor y de la conducta. Nuestro objetivo fue correlacionar el volumen de la lesión (en secuencias DRM y FLAIR) con puntuaciones directas en test cognitivos y afectivos. Se estudian 24 pacientes con lesiones vasculares cerebrales subcorticales, aplicando en dos ocasiones pruebas cognitivas y afectivas. Los resultados muestran una correlación negativa entre el volumen de la lesión en la secuencia DRM y las pruebas de fluencia verbal fonética $(r=-0,42)$ y denominación $(r=-0,64)$. También se observa una correlación negativa con la prueba de denominación de Boston $(r=-0,50)$ en la secuencia FLAIR. Según la lateralización de la lesión, resultan significativas las correlaciones entre el volumen de la misma en el hemisferio izquierdo y el TMT-B, y con el test de denominación. Con relación a la segunda evaluación llevada a cabo tres meses después, se observa una cierta mejoría de las puntuaciones directas.
\end{abstract}

Palabras clave: Ictus, lesiones subcorticales, evaluación, rendimiento cognitivo, afectividad.

\section{Cognitive function and affective symptoms during the acute phase and three months following an ischemic stroke}

\begin{abstract}
After an ictus, decline of cognitive function, mood and behaviour have been described. Our aim is to correlate the volume of the lesion (in sequences DRM and FLAIR) with the raw scores of cognitive and affective tests. Patients $(N=24)$ with vascular subcortical cerebral lesions were studied, and cognitive and affective behaviour tests were administered twice. The results show a negative correlation between the volume of the lesion in the DRM sequence and the verbal phonetic fluency $(r=-0,42)$ and naming scores $(r=-0,64)$. A negative correlation is also observed with the Naming Boston Test $(r=-0,50)$ in the FLAIR sequence. Correlations between the volume of the lesion in the left hemisphere and the Trail Making Test part B and the naming test, were statistically significant. With relationship to the second evaluation carried out after three months, a certain improvement of the raw scores of the tests was observed.
\end{abstract}

Keywords: Stroke, subcortical lesion, assessment, cognitive performance, mood.

\section{INTRODUCCIÓN}

En la fase aguda del ictus se ha descrito deterioro cognitivo, cambios de humor y de conducta (Pantoni y Garcia, 1996; Tatemichi et al., 1992; Méndez, Adams, y Lewandowski, 1989). Sin embargo, los aspectos cognitivos en la enfermedad cerebrovascular han permanecido frecuente-

Recibido: 23 julio 2007; aceptado 24 septiembre 2007

Correspondencia: Ángel Aguilar-Alonso, Departamento de Personalidad, Evaluación y Tratamiento Psicológicos, Pssg. Vall d'Hebrón, 171, 08035 Barcelona. Correo-e: aaguilaraguilarpsi@ub.edu mente ignorados, ya que el concepto de ictus hace referencia a los síntomas y signos focales que aparecen de inicio súbito. De esta forma, siempre se ha prestado más atención a la función motora y sensitiva, y menos a los déficit cognitivos de forma incipiente. Estas manifestaciones pueden persistir en el tiempo, dependiendo de la gravedad del ictus, localización hemisférica y estratégica de la lesión, tipo de infarto, número de infartos, recurrencias (Frank et al., 1996). La resonancia magnética (Rovira et al., 2000) permite observar lesiones en las primeras horas de la evolución del ictus isquémico. La secuencia de difusión (DRM) es una técnica con alta fiabilidad y sensibilidad (Sin- 
Tabla 1. Características de la muestra

\begin{tabular}{|c|c|c|c|}
\hline Variables & Porcentaje & Media & Desv. Típica \\
\hline Género (varones) & $71 \%$ & & \\
\hline Edad (años) & & 64 & 4,3 \\
\hline Educación (años) & & 8 & 4 \\
\hline Lesión (hemisferio izquierdo) & $45,8 \%$ & & \\
\hline Historia de trastorno de carácter & $8,3 \%$ & & \\
\hline Historia familiar de trastorno de carácter & $8,3 \%$ & & \\
\hline Ictus previo & $41 \%$ & & \\
\hline Unidad de Ictus & $41,7 \%$ & & \\
\hline Tiempo desde el ataque del ictus MRI (horas) & & 83,2 & 46,5 \\
\hline \multicolumn{4}{|l|}{ Tiempo (días) desde el establecimiento de los } \\
\hline síntomas hasta la evaluación neuropsicológica) & & 5,4 & 4,4 \\
\hline National Institute of Health Stroke Scale (NIHSS) & & 3,3 & 2,4 \\
\hline Barthel Index previo (BI) & $100 \%$ & & \\
\hline
\end{tabular}

ger et al., 1998) para detectar lesiones en la fase aguda del ictus isquémico (localización, extensión y número de lesiones) y, además, facilita la distinción de las lesiones crónicas de las que aparecen en fase aguda (Sunshine et al., 1999). Esto es importante, pues nos permite ver una lesión en la fase aguda, su evolución en las horas siguientes, y así poder cuantificar dicha lesión y relacionarla con las puntuaciones directas de los test que miden la función cognitiva y las escalas afectivas $\mathrm{y}$ de conducta.

Este estudio tiene como objetivo relacionar el volumen de las lesiones en las secuencias de resonancia magnética (difusión DRM y FLAIR), en la fase aguda del ictus, con las puntuaciones directas obtenidas por los pacientes en test cognitivos, afectivos y conductuales, tratando de verificar que a mayor volumen de la lesión corresponde un menor rendimiento cognitivo en las escalas correspondientes y una mayor puntuación en las escalas afectivas y conductuales de carácter patológico. También se pretende llevar a cabo un análisis de las diferencias en dichas puntuaciones de los test cognitivos y afectivos entre la fase aguda y tres meses después del ictus.

\section{MÉTODO}

\section{Participantes}

Se han estudiado 24 pacientes con lesiones vasculares cerebrales subcorticales. La edad media fue de $64(D T=4,3)$ años, con predominio del genero maculino ( $71 \%$ de la muestra). El nivel educativo en años fue de $8(D T=4)$. La lesión estaba localizada en el hemisferio izquierdo en 11 pacientes $(45,8 \%)$, en el hemisferio derecho 11 pacientes $(45,8 \%)$ y las lesiones eran bilaterales en 2 pacientes $(8,3 \%)$. Todos eran diestros y el $41 \%$ había sufrido un infarto anterior. Del total de la muestra, 10 $(41,7 \%)$ pacientes habían sido ingresados en la Unidad de Ictus, siendo monitorizados sus signos vitales durante la 72 horas posteriores a los síntomas neurológicos.

Las lesiones estaban localizadas en corona radiata $(n=9)$, en la cápsula interna $(n=3)$, en el tálamo $(\mathrm{n}=5)$, en el mesencéfalo y en la protuberancia $(n=1)$, en la protuberancia $(n=3)$, en el cerebelo $(n=2)$ y en cerebelo y la protuberancia $(n=1)$. Así pues, hay dos sujetos con doble lesión: uno en el mesencéfalo y la protuberancia y otro en el cerebelo y la protuberancia.

\section{Pruebas psicológicas}

Mini Mental State Examination (MMSE; Folstein, M.F., Folstein, S.E., y McHugh, 1975). Permite obtener el rendimiento cognitivo global. Consta de 30 ítem que evalúan la orientación temporo-espacial, memoria, atención y cálculo, denominación, repetición, lectura y escritura y copia de dibujos. 
Puebas de dígitos del WAIS (Dígitos O. Inverso y O. Directo) [Wechsler Intelligence Scale Subtest] (Lezak, 1983). Esta escala de la batería Wechsler evalúa la capacidad del sujeto para evocar series de dígitos progresivamente más largas, en el mismo orden y en orden inverso. Es una prueba estandarizada que se utiliza para valorar la atención/concentración y la memoria inmediata.

Fluencias fonéticas [PhF] y semánticas [SF] (Lezak, 1983). Esta prueba evalúa la producción espontánea de palabras. En la fluencia fonética se le pide al sujeto que emita palabras que comiencen por la palabra $(p)$. Se cuantifica el número de palabras que emite el sujeto en un minuto. En la fluencia semántica se le pide al sujeto que durante un minuto emita nombre de animales.

Boston Naming Test (BNT; Lezak, 1983). El Test de Denominación de Boston permite examinar la capacidad del sujeto para denominar objetos por confrontación visual. La prueba consta de 60 láminas y el sujeto ha de denominar correctamente los dibujos de todas ellas. La prueba se mide contando las láminas o dibujos acertados por el sujeto.

Trail Making Test (TMT-B; Lezak, 1983). Es una prueba estandarizada que se utiliza para valorar la velocidad psicomotora compleja, que depende de la preservación de la atención-concentración y de la función ejecutiva. Presenta dos partes: la parte (A) está formado por números, dispuestos de forma aleatoria dentro de un circulo, que van del 1 al 25 y que el sujeto ha de unir con un bolígrafo de forma consecutiva; la parte (B) está formada por números dispuestos de forma aleatoria dentro de un circulo que van del 1 al 13 y de letras que van de la A a la L. La tarea consiste en que el sujeto ha de unir los números y letras siguiendo el orden numérico y alfabético, alternando números y letras. En ambos test se valora el número de errores y el tiempo empleado por el sujeto.

Hamilton Depression Rating Scale (HRS-D; Hamilton, 1960). Se utiliza para observar posibles trastornos afectivos y para evaluar el estado de ánimo. Esta escala consta de 10 ítem y evalúa los siguientes aspectos: ánimo deprimido, sentimiento de culpabilidad, intento de suicidio, insomnio, perdida de interés para desa- rrollar tareas, lentitud de pensamiento, agitación, y ansiedad psíquica y somática.

Bech-Rafaelsen Manía Scale (BRMS; Bech, Bolwing, Kramp, y Rafaelsen, 1979). Se utiliza para detectar procesos hipomaníacos. Esta escala evalúa los siguientes ítem: la actividad motora, fuga de ideas, intensidad del tono de voz, estado de ánimo elevado, sentimiento de autoestima, contacto verbal, sueño, actividad sexual $\mathrm{y}$ trabajo e intereses.

Maudsley Obsesive-Compulsive Inventory (MOCI; Hodgson, y Rachman, 1977). Los 30 «ítem» de este inventario permiten obtener una determinada puntuación sobre las ideas y actos compulsivos del sujeto, que, en el presente trabajo se administra después de haber sufrido un ictus. Concretamente, la escala está dividida en cuatro sub-escalas que miden procesos de comprobación, limpieza, lentitud y repetición, y la duda y conciencia del sujeto

National Institute Health Stroke Scale (NIHSS; Goldstein, Bertels, Davis, 1989). Es una escala del Instituto Nacional de Salud de los EEUU para valorar la extensión del infarto cerebral. La escala de evaluación del infarto cerebral fue diseñada sólo para AVC isquémicos, pero puede ser útil en todos los casos de AVC. El rango va de 0 puntos (normal) a 42 puntos (COMA). Los puntajes de más de 20 son más vulnerables a transformación hemorrágica y están asociados a un pobre diagnóstico.

Índice de Barthel (IB; Shah, Vanclay, y Cooper, 1989). Este valora el nivel de independencia del paciente con respecto a la realización de algunas actividades de la vida diaria (AVD), mediante la cual se asignan diferentes puntuaciones según la capacidad del sujeto. Las AVD incluidas en este test evalúan: si el sujeto puede comer solo, trasladarse de la silla a la cama, aseo personal, uso del retrete, bañarse/duchase solo, desplazarse por superficies lisas o en silla de ruedas, subir o bajar escaleras, vestirse o desvestirse, control de orina. Estas actividades se puntúan de 0 a 100 puntos.

\section{Procedimiento}

Los pacientes fueron incorporados al estudio después de su ingreso en el Servicio de Neuro- 
logía del Hospital de Girona. En el momento del ingreso, todos presentaban signos y síntomas neurológicos y cognitivos, por lo que fueron evaluados por un neurólogo, quien, después de un proceso de valoración, los ingresaba en la planta de neurología, unidad de ictus o habitación convencional. Todos los pacientes fueron informados del proceso de la investigación y firmaron la correspondiente solicitud de consentimiento informado. Posteriormente fueron evaluados mediante una TAC para descartar hemorragia y, en las 48 horas siguientes, se les practicó una resonancia magnética, secuencias de difusión (DRM) y FLAIR. El tiempo transcurrido entre los síntomas clínicos y la resonancia magnética fue de 83,2 $(D T=46,5)$ horas. En el transcurso de las dos primeras semanas, los pacientes informaron de su historia individual y familiar de posible trastorno mental; variable que podía afectar al estudio. Dos sujetos $(8,3 \%)$ de la muestra habían presentado depresión en algún momento de su vida, y otros dos $(8,3 \%)$ tenían parientes directos que habían padecido algún trastorno depresivo. Ninguno de los pacientes de la muestra cumplía criterios de abuso o dependencia de alcohol y drogas, ni de demencia, según el DSM-IV (APA, 2000). Seguidamente les fueron administradas las puebas de evaluación indicadas.

Las pruebas anteriores se administraron teniendo en cuenta la fatiga del paciente, de forma que a algunos les fueron adminstradas en dos días succesivos, si su estado lo requería. El tiempo transcurrido entre el comienzo de los síntomas neurológicos y el inicio de la evaluación psicológica fue de una media de 5,4 (DT $=4,4)$ días.

En relación con el diagnóstico y medición del volumen de las lesiones cerebrales se efectuó como se indica a continuación. La técnica de neuroimagen (TAC) fue llevada a cabo en las primeras horas del inicio de los síntomas neurológicos para descartar hemorragia y, posteriormente, una resonancia magnética para determinar el alcance de la lesión. La resonancia magnética (RM) se realizó con un equipo de 1,5 Teslas con secuencias eco-planar (Philips.Giroscan). El examen incluyó las siguientes secuencias: Axial T2-FLAIR, Axial GE, Axial Difusión. Los pacientes que tenían lesio- nes corticales en la fase aguda fueron excluidos del estudio. Sólo fueron incluidos aquellos pacientes en los cuales se observó una lesión subcortical en la fase aguda del ictus (corona radiata, ganglios de la base, tálamo, mesencéfalo, protuberancia y cerebelo) en las dos secuencias: difusión (DRM) y FLAIR. Las imágenes obtenidas por RM fueron evaluadas por un neurorradiólogo independiente, desconociendo los resultados de la evolución clínica y de los datos de laboratorio.

En la secuencia de difusión por RM se escogieron las imágenes con posible infarto, para la obtención de las imágenes TRACE resultado de la sumación de las imágenes del mismo nivel. El volumen de la lesión fue determinado mediante las imágenes de difusión. El mapa de ADC se obtuvo a partir de las imágenes de difusión, cuantificando el ADC en la zona isquémica, con lo cual fueron determinados la localización y el tamaño de la lesión isquémica, así como el tiempo de la evolución. Posteriormente, las imágenes de cada estudio fueron enviados a una estación de trabajo, en cuyo ordenador se disponía de un programa informático para permitir su análisis. Cada lesión focal fue analizada por el método planimétrico. En cada corte se subrayó el borde de la lesión de modo manual, consiguiendo así el valor del área de la lesión. A continuación se multiplicó por el grosor del corte y se obtuvo el volumen de ese segmento de la lesión. Repitiendo la operación en todos los cortes, y sumando todos los volúmenes de las secciones, se obtuvo el volumen total de la lesión.

\section{Análisis estadístico}

Para calcular las posibles correlaciones entre el volumen de las lesiones y el rendimiento cognitivo y afectivo de los pacientes, teniendo en cuenta lo reducido de la muestra, se utilizó la técnica no paramétrica Rho de Spearman. Para el análisis de estadístico de las diferencias entre las puntuaciones directas en las diferentes pruebas cognitivas, afectivas y conductuales, también se aplican técnicas no paramétricas como la U-Mann-Whitney, para la verificación de los posibles niveles de sig- 
nificación $(p<0.05)$, así como para comprobar las posibles diferencias por la localización de la lesión.

\section{RESULTADOS}

El volumen medio de las lesiones en DRM fue de $0,89(D T=1,2) \mathrm{cm}^{3}$, y en FLAIR de $0,88(D T=1,1) \mathrm{cm}^{3}$, con una diferencia significativa $(p<0,001)$ entre los volúmenes de ambas secuencias.

En la Tabla 2, podemos ver que, en la secuencia de difusión (DWI), el volumen de las lesiones correlaciona negativa y significativamente con las puntuaciones directas del rendimiento cognitivo en las pruebas de fluencia fonética y con el test de denominación BNT $(r=-0,421$ y $r=-0,64)$, de forma que a mayor volumen se da un menor rendimiento y viceversa. En la secuencia FLAIR, el volumen de las lesiones correlaciona significativamente con el test de denominación BNT $(r=-0,50)$. Las correlaciones no alcanzan la significación estadística con las otras pruebas: Dígitos, fluencia semántica y TMT-B. Tampoco se obtienen correlaciones significativas entre el volumen de las lesiones y las puntuaciones directas en las escalas afectivas HRSD y BRMS, aunque la correlación del inventario de obsesiones-compulsiones MOCI es alta y positiva $(r=0,48)$ en la secuencia FLAIR.
Tabla 2. Correlaciones no paramétricas (Rho de Spearman) entre los volúmenes de las lesiones DWI y FLAIR y las puntuaciones directas en los test cognitivos y afectivos

\begin{tabular}{lcc}
\hline & $\begin{array}{c}\text { Secuencia } \\
\text { DWI }\end{array}$ & $\begin{array}{c}\text { Secuencia } \\
\text { FLAIR }\end{array}$ \\
\hline MMSE & $-0,24$ & 0,07 \\
Dígitos (O. inverso) & 0,05 & 0,028 \\
Dígitos (O. directo) & $-0,21$ & $-0,15$ \\
PhF & $-0,42^{*}$ & $-0,37$ \\
SF & $-0,22$ & $-0,26$ \\
BNT & $-0,64^{* *}$ & $-0,50^{*}$ \\
TMT-B (segundos) & 0,34 & 0,29 \\
HRS-D & $-0,16$ & $-0,24$ \\
BRMS & $-0,36$ & $-0,33$ \\
MOCI & 0,20 & 0,48 \\
\hline
\end{tabular}

MMSE: Mini Mental State Examination; Dígitos (orden inverso): Dígitos (orden directo) WAIS; PhF: Fluencia fonética; SF: fluencia semántica; BNT: Test de Denominación de Boston; TMT-B: Trail Making Test (B); HRS-D: Escala de Depresión de Hamilton; BRMS: Escala de Manía de Bech-Rafaelsen; MOCI: Maudsley ObsesiveCompulsive Inventory. ${ }^{*} p<0,01 ;{ }^{* *} p<0,001$.

Cuando las correlaciones se calculan separando a los pacientes en dos grupos por la lateralización hemisférica de la lesión (izquierda/derecha), podemos observar en la Tabla 3 que en los pacientes con lesiones en el hemisferio izquierdo en la secuencia DWI, el volumen de la lesión correlaciona negativamente

Tabla 3. Correlaciones no paramétricas (Rho de Spearman) entre los volúmenes de las lesiones DWI y FLAIR, y las puntuaciones directas en los test cognitivos y afectivos, según la lateralidad del hemisferio afectado, sin tener en cuenta los bilaterales

\begin{tabular}{lcccc}
\hline & $\begin{array}{c}\text { Hemisf. derecho } \\
(n=11)\end{array}$ & $\begin{array}{c}\text { Hemisf. derecho } \\
(n=11)\end{array}$ & $\begin{array}{c}\text { Hemisf. izquierdo } \\
(n=11)\end{array}$ & $\begin{array}{c}\text { Hemisf. izquierdo } \\
(n=11)\end{array}$ \\
\cline { 2 - 5 } & Secuencia DWI & Secuencia FLAIR & Secuencia DWI & Secuencia FLAIR \\
\hline MMSE & 0,55 & $0,85^{* *}$ & $-0,58^{*}$ & $-0,45$ \\
Dígitos (O. inverso) & 0,45 & $-0,07$ & 0,01 & 0,17 \\
Dígitos (O. directo) & 0,15 & $-0,35$ & $-0,38$ & $-0,31$ \\
PhF & $-0,18$ & $-0,51$ & $-0,09$ & $-0,02$ \\
SF & $-0,13$ & $-0,20$ & $-0,11$ & $-0,23$ \\
BNT & $-0,31$ & $-0,24$ & $-0,68^{*}$ & $-0,48$ \\
TMT-B & $-0,28$ & $-0,39$ & $0,50 *(n=8)$ & $0,67 *(n=8)$ \\
HRS-D & $-0,41$ & $-0,43$ & $-0,12$ & $-0,19$ \\
BRMS & $-0,33$ & $-0,11$ & $-0,09$ & $-0,12$ \\
MOCI & 0,45 & 0,49 & 0,11 & 0,18 \\
\hline
\end{tabular}

$* p<0,05 ; * *<0,001$. 
con el test de denominación BNT $(r=-0,67)$, de forma que a un mayor volumen le corresponde un peor rendimiento, y positivamente con el TMT-B $(r=0,50)$, es decir, un menor rendimiento, ya que a mayor volumen se da un mayor tiempo en segundos al realizar esta tarea. En la secuencia FLAIR, este mismo test de la función ejecutiva aumenta su correlación con el volumen $(r=0,667 ; p<0,01)$, a pesar del bajo número de pacientes $(\mathrm{n}=8)$. De las escalas afectivas, ninguna resulta significativa.

En el grupo de sujetos con lesiones en el hemisferio derecho, sólo aparece una correlación positiva y altamente significativa en la secuencia FLAIR entre el rendimiento global medido con el Minimental (MMSE) y el volumen $(r=, 852)$. Es de difícil explicación, por cuanto que implica que a mayor lesión, se da un mejor rendimiento cognitivo general, lo que probablemente corresponde a características particulares de la escasa muestra $(n=11)$. No alcanzan la significación estadística las correlaciones del inventario MOCI en ambas secuencias, aunque son altas y positivas: tiene sentido que este tipo de lesiones venga acompañado de un mayor número de síntomas de obsesióncompulsión.

Cuando se comparan las medias de las puntuaciones en los test cognitivos y afectivos obtenidas por los pacientes en la fase aguda del ictus subcortical con las obtenidas tres meses después, se observan diferencias estadística- mente significativas en los test de fluencia semántica SF $(p<0,05)$, Boston Naming Test BNT $(p<0,01)$, Bech-Rafaelsen Mania Scale (BRMS) $(p<0,01)$ y Maudlsey ObsessiveCompulsive Inventory (MOCI) $(p<0,01)$ (véase la Tabla 4)-

Cuando esta misma comparación se realiza en una submuestra de sujetos en los que la lesión se localiza en el hemisferio izquierdo, se observan diferencias significativas en los siguientes tests: Dígitos (orden directo), SF y TMT-B. Así pues, tres meses después del ictus, se da una mejoría del rendimiento, con un aumento estadísticamente significativo de la media en dígitos y fluencia semántica y un menor tiempo en segundos en la tarea de trazo del TMT-B. Cuando la comparación se realiza en la submuestra de sujetos cuyas lesiones se localizan en el hemisferio derecho, se observan diferencias significativas en los test de denominación BNT, la escala BRMS y el inventario MOCI, de forma que se da una significativo aumento de la media en la capacidad de denominación y una disminución de los items de manía y de obsesiones-compulsiones. (Véase la Tabla 5).

\section{DISCUSIÓN Y CONCLUSIONES}

En el estudio que hemos realizado con las secuencias de DRM y FLAIR, se observa que

Tabla 4. Puntuaciones directas en los test cognitivos y afectivos en la fase aguda y tres meses después del ictus.

\begin{tabular}{|c|c|c|c|}
\hline \multirow[b]{3}{*}{ MMSE } & \multirow{2}{*}{$\begin{array}{c}\text { Fase aguda } \\
\text { Media }(D T) \\
(n=24)\end{array}$} & \multicolumn{2}{|l|}{ Tres meses } \\
\hline & & $\begin{array}{l}\text { Media }(D T) \\
\quad(n=22)\end{array}$ & Sig. (diferencia) \\
\hline & $27,2 \quad(2,8)$ & $(1,6)$ & 0,128 \\
\hline Dígitos (O. inverso)) & $(0,8)$ & $3,6 \quad(0,9)$ & 0,137 \\
\hline Dígitos (O. directo) & $(0,8)$ & $(0,8)$ & 0,451 \\
\hline $\mathrm{PhF}$ & $7,7 \quad(4,6)$ & $8,1 \quad(4,5)$ & 0,550 \\
\hline SF & $(3,5)$ & $(3,4)$ & $0,015^{*}$ \\
\hline BNT & $46,3 \quad(7,2)$ & $47,5 \quad(6,6)$ & $0,005^{*}$ \\
\hline TMT-B (sec) & $213,3(113,3)$ & $203,6(103,6)$ & 0,546 \\
\hline HRS-D & $3,2 \quad(3,3)$ & $2,6 \quad(3,4)$ & 0,381 \\
\hline BRMS & $1,4 \quad(1,9)$ & $0,4 \quad(1,1)$ & $0,002 *$ \\
\hline MOCI & $13,22 \quad(4,26)$ & $10,89 \quad(4,2)$ & $0,002 *$ \\
\hline
\end{tabular}

$* p<0,05$. 
Tabla 5. Puntuaciones directas en los test cognitivos y afectivos en la fase aguda y tres meses después del ictus, en submuestras de los sujetos clasificados según la lateralidad de la lesión.

\begin{tabular}{lcccc}
\hline & Fase aguda & 3 meses & Fase aguda & 3 meses \\
& H. izquierdo & H. izquierdo & H. derecho & H. derecho \\
\cline { 2 - 5 } & Media $(D T)$ & Media $(D T)$ & Media $(D T)$ & $\begin{array}{c}\text { Media }(D T) \\
(n=10)\end{array}$ \\
& $(n=10)$ & $(n=10)$ & $28,3(1,2)$ & $28,4(1,1)$ \\
MMSE & $26,3(3,7)$ & $27,9(2,0)$ & $4,9(0,9)$ & $5(0,9)$ \\
Dígitos (O. directo) & $4,5(0,8)$ & $4,9(0,9)^{*}$ & $3,2(0,8)$ & $3,2(0,8)$ \\
Dígitos (O. inverso) & $3,6(0,9)$ & $3,8(0,9)$ & $8,8(3,5)$ & $8,7(4,2)$ \\
PhF & $7,1(5,8)$ & $7,3(5,2)$ & $12,4(4,1)$ & $14,9(2,7)$ \\
SF & $12,3(3,1)$ & $4,3(4,4)^{*}$ & $47,9(6,3)$ & $48,9(6,0)^{*}$ \\
BNT & $44,6(8,1)$ & $179,1(119,3)^{*}$ & $202,1(84,1)$ & $225,2(87,9)$ \\
TMT-B (segundos) & $223,1(153,6)$ & $2,0(2,5)$ & $3,6(3,6)$ & $3,3(4,4)$ \\
HRS-D & $2,5(3,2)$ & $0,0(0,0)$ & $2,2(2,2)$ & $0,8(1,6)^{*}$ \\
BRMS & $0,6(1,3)$ & $12,4(5,2)$ & $11,9(3,8)$ & $9,8(3,6)^{*}$ \\
MOCI & $14,8(5,2)$ & & & $9,8)$ \\
\hline
\end{tabular}

$* p<0,05$.

el aumento del volumen de la lesión está interfiriendo en el rendimiento cognitivo de los pacientes en pruebas que miden lenguaje, atención y funciones ejecutivas. Moser et al. (2001) ya han relacionado las lesiones hiperintensas subcorticales en MRI con la función ejecutiva en pacientes con demencia vascular, encontrando relaciones estadísticas significativas entre el enlentecimiento cognitivo, déficit de atención y las manifestaciones conductuales. Los infartos lacunares, por sí solos, no producen demencia, pero estas lesiones pueden interferir en el rendimiento cognitivo, sobre todo cuando las lesiones están localizadas en el núcleo caudado (Hirozaku Bokura, y Robinson, 1997; Kumral, Evyapan, y Balkir, 1999), en el tálamo (bilateral y núcleos que irrigan la arteria tuberotalámica y paramediana) (Gutiérrez et al., 2003; Schmahmann, 2003), o cuando las lesiones afectan a la sustancia blanca profunda (Ylikoski et al., 1993; De Groot et al., 2002), lo que provoca un enlentecimiento cognitivo, alteraciones de la memoria, déficit de atención, alteraciones del lenguaje, alteración de las funciones ejecutivas y cambios de personalidad. En otros estudios (Henon, Pasquier, Durieu, Pruvo, y Leys, 1998), se ha observado que la atrofia cerebral correlaciona con el deterioro cognitivo severo (demencia), sobre todo si la atrofia es de predominio temporal. Además, también hay estudios (Del Ser et al., 1990) que han observado que el deterioro cognitivo está relacionado con el número de infartos lacunares, por lo que ha sido denominado el estado lacunar. También se han podido observar alteraciones del lenguaje en lesiones subcorticales, así Nadeau y Crosson (1997) observaron afectaciones de la fluencia, repetición, denominación, comprensión y errores parafásicos en pacientes con lesiones en la cabeza del núcleo caudado, putamen y miembro anterior de la cápsula interna. Los trastornos afásicos, generalmente han estado asociados a lesiones corticales, pero Hillis y sus colaboradores (Hillis et al., 2002) han demostrado en sus estudios con resonancia magnética (secuencia de difusión DWI y perfusión PWI), que en la fase aguda del ictus isquémico, cuando se observa una lesión subcortical en las primeras horas en difusión (DWI), con afectación del lenguaje, en la mayoría de pacientes, también se puede observar en la secuencia de perfusión una zona de penumbra isquémica que sobrepasa la propia lesión estructural y afecta a zonas corticales.

En nuestro estudio de pacientes con lesiones subcorticales, encontramos que en aquellos que manifiestan dichas lesiones en el hemisferio izquierdo, según las secuencias DWI y FLAIR, el volumen de las lesiones correlaciona de forma positiva y estadísticamente significativa con las puntuaciones obtenidas en el TMT-B. Dado que este test está diseñado para medir alteracio- 
nes de la atención/concentración, capacidad visuomotora, organización de la información y función ejecutiva, nos lleva a pensar que el aumento del volumen de las lesiones localizadas en el hemisferio izquierdo está deteriorando las funciones de atención/concentración, organización de la información y ejecutivas en estos pacientes, y parece que no ocurre lo mismo con los que las manifiestan en el hemisferio derecho. Además, la correlación entre el volumen de la lesión y el test TMT-B (Tabla 3) se da en ambas secuencias de resonancia magnética (DRM y FLAIR) por lo que podemos pensar que el efecto del ictus sobre la tarea de trazo no se produce solamente en la fase aguda, sino que puede ser una función cognitiva alterada de forma permanente en estos pacientes. Estos resultados, junto a las significativas correlaciones negativas entre los test de denominación de Boston y el test de rendimiento cognitivo global MMSE con el volumen de la lesión en el hemisferio izquierdo en la secuencia DWI, nos recuerdan los del estudio de Langdon y Warington (2000), quienes con una muestra de 40 pacientes con lesiones isquémicas unilaterales en los hemisferis izquierdo y derecho, evaluando tareas verbales, espaciales y procesos de razonamiento, concluyen que el hemisferio izquierdo es crucial para el procesamiento verbal, espacial y el razonamiento abstracto.

Hay autores (Lawrence et al., 1999) que han observado, en pacientes con infartos focales isquémicos subcorticales, una disminución del flujo sanguíneo cerebral (hipometabolismo) en áreas del lóbulo frontal usando tomografía por emisión de positrones simples. (PET). Las lesiones en territorio subcortical pueden afectar a la cognición a través de desconexión de las vías cortico-subcorticales (Cummings, 1993; Alexander, DeLong, y Strick, 1986). Por otro lado, no sabemos con exactitud cuál es la forma en la que una lesión de origen vascular destruye la estructura anatómico-funcional que da sustento a las funciones cognitivas. Como ha postulado Mesulan (1985), el funcionamiento cerebral puede estar asentado en redes neuronales de poblaciones celulares corticales y núcleos grises subcorticales que se conectan entre sí mediante fibras que viajan por la sus- tancia blanca (Martínez-Lage, y Hachinski, 1998). Por lo tanto, el volumen y la localización de la lesión isquémica son importantes para valorar las funciones cognitivas afectadas.

De nuestro trabajo, queda por explicar la extraña correlación positiva, estadísticamente significativa entre las puntuaciones en el test Minimental y el volumen de las lesiones del hemisferio derecho. Sólo cabe interpretarlas en función del azar, como consecuencia de particularidades de la escasa muestra. Sin embargo resulta lógica la ligera mejoría de los pacientes en su puntuaciones medias en los test cognitivos después de los tres meses del ictus, aunque sólo resulten estadísticamente significativas las pruebas de fluencia semántica y la de denominación de Boston en la muestra global, los dígitos en orden directo y el tiempo en el test TMT$\mathrm{B}$ en la submuestra de los sujetos con lesiones en el hemisferio izquierdo, y el test de denominación de Boston en los sujetos con lesiones en el hemisferio derecho.

Con referencia a los síntomas afectivos de nuestra muestra de pacientes, el volumen de las lesiones subcorticales no correlaciona significativamente con las puntuaciones directas en las escalas de depresión HRS-D y manía BRMS (Tabla 3). En cuanto a los síntomas obesivo-compulsivos, aunque las correlaciones positivas del MOCI parecen altas, especialmente en el caso del hemisferio derecho en ambas secuencias (DWI $r=0,45$; FLAIR $r=0,49$ ), no alcanzan la significación estadística, probablemente por el escaso número de sujetos, dado que hubo de eliminar de la muestra a los dos sujetos con lesiones bilaterales.

En cuanto a la esperada mejoría entre las puntuaciones medias directas obtenidas en la fase aguda del ictus y la evaluación posterior tres meses más tarde, se observan diferencias estadísticas en la escala BRMS; $p<0,01)$ y en el inventario MOCI $(p<0,01)$, de forma que se da una significativa disminución de los síntomas de manía y obsesiones-compulsiones. Sin embargo la ligera disminución observada en la escala HRS-D no resulta estadísticamente significativa (Tabla 4). Starkstein, Robinson, Berthier, Parisk, Price et al. (1988) examinaron pacientes con infartos vasculares, lesiones unilaterales en ganglios basales y tálamo observa- 
das por CT scan y en presencia de trastornos del humor, y concluyeron que los pacientes con lesiones izquierdas en la cabeza del núcleo caudado muestran una alta frecuencia de síntomas depresivos comparados con pacientes con lesiones en hemisferio derecho e incluso comparadas con otras lesiones del hemisferio izquierdo. Ellos sugirieron que las proyecciones frontocaudadas pueden jugar un papel importante en la modulación de los trastornos del humor. Por su parte, Morris, Robinson, Raphael, Hopwood, et al., 1996), evaluaron una muestra de 41 pacientes con lesiones isquémicas focales observados en CT scan y la presencia de síntomas depresivos, y concluyeron que los sujetos con lesiones que envuelven el lóbulo prefrontal y ganglios basales izquierdos tienen más frecuentemente síntomas depresivos que los pacientes con lesiones en el hemisferio derecho. En nuestra muestra, no se observan síntomas depresivos severos en la fase aguda, y como las puntuaciones del conjunto de las escalas afectivas mejoran tres meses depués, esto puede indicar que los síntomas depresivos leves que se detectan están asociados a la fase aguda del ictus y a los estímulos ambientales que se desencadenan, más que a la propia lesión producida por el infarto isquémico subcortical.

En cuanto a los procesos hipomaníacos, generalmente son asociados a un amplio grupo heterogéneo de desordenes neurológicos (Shulman, Tohen, Satlin, Mallya, y Kalunian, 1992). Así, los pacientes con lesiones en el hemisferio derecho, sobretodo en regiones orbitofrontales y temporales, parecen ser más proclives a procesos hipomaniacos (Strakowski, McElroy, Keck, y West, 1994). Hay autores como Cummings (1993) y Shulman y Hermann (1999) que han sugerido que, para que se dé un carácter normal, es precisa la correcta función de los circuitos que integran los sistemas frontal, límbico y ganglios basales.

La valoración de los trastornos cognitivoconductuales en nuestra muestra mediante el MOCI se lleva a cabo prque no faltan autores (McGuire, 1995; Berthier, Kulisevsky, Gironell, 1996) que han asociado las lesiones estructurales cerebrales (un 20\% aproximadamente) que afectan mayoritariamente a los ganglios basales, lóbulo temporal y frontal, con una mayor producción de actos obsesivos y compulsivos. En nuestro trabajo, el volumen de las lesiones no correlaciona significativamente con las puntuaciones directas de la escala MOCI, ni en la muestra total de pacientes ni separados por la localización hemisférica (Tablas 2 y 3 ), aunque, como hemos visto, en el caso del hemisferio derecho, en las dos secuencias, sin tener en cuenta los casos con lesiones bilaterales, las correlaciones son altas $(r=0,45$ y $r=0,49)$ y cercanas a la significación.

Las lesiones isquémicas subcorticales producen en la fase aguda síntomas que pueden confundirse con estados de ánimo depresivo, pues se altera el sueño (insomnio), agitación, ansiedad y síntomas somáticos. Como se ha descrito en la literatura científica especializada, los trastornos afectivos (especialmente síntomas depresivos) están más asociados al núcleo caudado y conexiones con el lóbulo prefrontal y basolateral. En nuestro estudio no obtuvimos pacientes con lesiones en el núcleo caudado, y los pacientes con lesiones corticales eran excluidos del estudio. Por lo tanto, pensamos que, quizás, sea esta la razón por la cual no se obtengan puntuaciones altas en la escala de depresión. Sim embargo, lo que sí resulta curioso es que cuando se hacen dos grupos de pacientes según su puntuación directa en el MOCI ( $\leq 12$ y ?13), los de la puntuación superior también tienen una significativa mayor media en la escala de depresión HRS-D ( $p<$ $0,05)$. La explicación podría ser discutida en base a las creencias disfuncionales asociadas a los síntomas obsesivo-compulsivos (Giménez, Morillo, Belloch, Carrió, y Cabedo, 2004). Aunque una severa autocrítica nos debería hacer reflexionar sobre el uso y abuso de los autoinformes en evaluación (Fernández-Montalvo y Echeburúa, 2006).

En cualquier caso, la conclusión más general de nuestro trabajo es que los infartos isquémicos focales de pequeño tamaño en estructuras subcorticales en fase aguda afectan a la cognición. Por ello es recomendable hacer un seguimiento de las funciones cerebrales superiores en este tipo de pacientes, pues además de que exista el riesgo de que en un futuro puedan desarrollar una demencia, en cualquier caso, el deterioro cognitivo de algunas funciones cog- 
nitivas (lenguaje, memoria, atención, ejecutivas, etc.) puede afectar a la rehabilitación a posteriori y a las actividades de la vida diaria.

\section{REFERENCIAS BIBLIOGRAFÍCAS}

Alexander, G.E., DeLong, M.R., y Strick, P.1. (1986). Parallel organization of functionally segregated circuits linking basal ganglia and cortex. Annual Review of Neuroscence, 9, 357-381.

American Psychiatric Association (2000). DSM-IV-TR Diagnostic and Statistical Manual of Mental Disorders, Fourth Edition, Text Revision. Washington \& London: Author.

Bech, P., Bolwing, T.G., Kramp, P., y Rafaelsen, O.J. (1979). The Bech-Rafaelsen Mania Scale and the Hamilton Depression Scale. Acta Psychiatrica Scandinavica, 59, 420-430.

Berthier, M.L., Kulisevsky, J., Gironell, A., y Heras, J.A. (1996). Obsessive-compulsive disorder associated with brain lesions. Clinical phenomenology, cognitive function, and anatomic correlates. Neurology, 47, 353-361.

Boiten, J., y Lodder, J. (1991). Lacunar infarcts: Pathogenesis and validity of the clinical syndromes. Stroke, 22, 1374-1378.

Cummings, J.L. (1993). Frontal-subcortical circuits and human behavior. Archives of Neurology, 50, 873-880.

De Groot, J.C., De Leeuw, F.E., Oudkerk, M., Van Gijn, Hofman, A., y Jolles, J. (2002). Periventricular cerebral white matter lesions predict of cognitive decline. Annals of Neurology, 52, 335-41.

Del Ser, T., Bermejo, F., Portera, A., Arredondo, J.M., Bouras, C., Constantinidis, J., et al. (1990). Vascular dementia. A clinicapathology strudy. Journal of the Neurological Sciences, 96, 1-17.

Fazekas, F., Ropele, S., Bammer, R., Kapeller, P., Stollberger, R., y Schmidt, R. (2000). Novel imaging technologies in the assessment of cerebral ageing and vascualr dementia. Journal of Neural Transmision, Supp. 59, 45-52.

Fernández-Montalvo, J., y Echeburúa, E. (2006). Uso y abuso de los autoinformes en la evaluación de los trastornos de personalidad. Revista de Psicopatología y Psicología Clínica, 11, 1-12.

Folstein, M.F., Folstein, S.E., y McHugh, P.R. (1975). «Mini Mental State». A practical method for grading the cognitive state of patients for the clinician. Journal of Psychiatric Research, 12,189-98.

Frank, A., Pohjasvaara, T., Erkinjunttin, T., Rarreiro, Tella, P., y Díez-Tejedor, E. (1996). Trastornos cognitivos relacionados con la isquémia cerebral. Revista Clinica Española, 196, 51-61.
Giménez, A., Morillo, C, Belloch, A., Carrió, C., y Cabedo, E. (2004). Creencias disfuncionales asociadas a síntomas obsesivo-compulsivos: una aproximación a la especificidad. Revista de Psicopatología y Psicología Clínica, 9, 13-20.

Goldstein, L.B., Bertels, C., y Davis, J.N. (1989). Interrater reliability of the NIH Stroke Scale. Archives of Neurology, 46, 660-662.

Gutiérrez, L., Dávalos, A., Pedraza, S., García-Sánchez, C., y Kulisevsky, J. (2003). Neuropsychological and behavioral impairment resulting from bilateral thalamic infart. Neurologia, 18, 404-408.

Hamilton, M.A.: (1960). A rating scale for depression. Journal of Neurology Neurosurgery and Psychiatry, 23, 56-62.

Henon, H.. Pasquier, F., Durieu, I., Pruvo, J.P., y Leys, D. (1998). Medial temporal lobe atrophy in stroke patiens: Relation to pre-existing dementia. Journal Neurology Neurosurgery and Psychiatry, 65, 641-647.

Hillis, A.E., Wityk, R.J., Barker, P.B., Beauchamp, N.J., Gailloud, P., Murphy, K., et al. (2002). Subcortical aphasia and neglect in acute stroke: The role of cortical hypoperfusion. Brain, 125, 1094-1104.

Hirozaku Bokura, y Robinson, R.G. (1997). Long-term cognitive impairment associated with caudate stroke. Stroke, 28, 970-975.

Hodgson, R.J., y Rachman, S. (1977). Obsessional-Compulsive Complaints. Behaviour Research and Therapy, 5, 389-395. Versión española de la Sección de Neurología del Hospital de la Santa Creu i Sant Pau de Barcelona.

Kumral, E., Evyapan, D., y Balkir, K.(1999). Acute caudate vascular lesions. Stroke, 30, 100-108.

Langdon, D., y Warington, E.K. (2000). The role of the left hemisphere in verbal and spatial reasoning tasks. Cortex, 36, 691-702.

Lawrence, T., Kwan, B.S., Bruce, R., Eberling, J.L., Schuff, N., Tanabe, J., et al. (1999). Effect of subcortical cerebral infarctions on cortical glucose metabolism and cognitive function. Archives of Neurology, 56, 809-814.

Lezak, M.D. (1983). Neuropsychological assessment (2nd ed.). New York: Oxford University Press.

Martínez-Lage, y Hachinski, V.C. (1998). Multi-infarct dementia. The vascular causes of cognitive impairment and dementia. En H.J.M., Barnet, J.P Mohr., BM Stein, y FM, Yatsu (eds.), Stroke: Pathophysiology, diagnosis, and management. 3rd Edition (pp. 875-894). New York: Churchill Livinstone.

McGuire, P.K. (1995). The brain in obsessive-compulsive disorder. Journal Neurology Neurosurgery and Psychiatry, 59, 457-459.

Méndez, M.F., Adams, N.L., y Lewandowski, KS. (1989). Neurobehavioural changes associated with caudate lesions. Neurology, 39, 349-354. 
Mesulan, M. (1985). Principles of Behavioral Neurology. Filadelfia: David Co.

Morris, P.1., Robinson, R.G., Raphael, B., y Hopwood, M.J. (1996). Lesion location and post-stroke depression. Journal of Neuropsychiatry and Clinical Neurosciences , 8, 399-403.

Moser, D.J., Cohen, R.A., Paul, R.H., Paulsen, J.S., Ott, B.R., et al. (2001). Executive functions and magnetic resonance imaging subcortical hyperintensities in vascular dementia. Neuropsychiatric Neuropsychology, and Behavioural Neurology, 14, 89-92.

Nadeau, S.E., y Crosson, B. (1997). Subcortical aphasia. Brain and Language, 58, 355-402.

Pantoni, L., García JH. (1996). Cerebral ischemia and cognitive impairment in the elderly. Facts and Research in Gerentology. Stroke (suppl.), 171-182.

Rovira, A., Pedraza, S., Molina, C., Capellades, J., Crivé, E., Rovira, A., y Montaner, J. (2000). Difusión por resonancia magnética en el diagnóstico de los infartos subcorticales agudos. Revista de Neurología, 30, 914919.

Shah, S., Vanclay, F., y Cooper, B. (1989). Improving the sensitivity of the Barthel Index for stroke rehabilitation. Journal of Clinical Epidemiology, 42, 703-709.

Singer, M.B., Chong, J., Lu D, Schonville, W.J., Tuhrim, S., y Atlas, S.W.(1998). Diffusion-weighted MRI in acute subcortical infarction. Stroke, 29, 133-136.

Sunshine, J.L., Tarr, R.W.,Lanzier, C.F., Landis, D.M.D., Selman, W.R., y Lewin, J.S. (1999). Hyperacute stro- ke: Ultrafast MR imaging to triage patients prior to therapy. Radiology, 29, 631-7.

Tatemichi, T.K., Desmond, D.W., Prohovnik, I., Cross, D.T., Gropen, T.I., Mohr, J.P., et al. (1992). Confusion and memory loss from capsualar genu infarction: A thalamocortical disconection syndrome? Neurology, 42, 1966-1979.

Schamahmann, G.D.(2003). Vascular syndromes of the thalamus. Stroke, 34, 2264-2278.

Shulman, K., Tohen, M., Satlin, A., Mallya, G., y Kalunian, D. (1992). Mania compared to unipolar depression in old age. American Journal of Psychiatry, 149, $341-5$.

Shulman, K.L., y Herrmann, N. (1999). The nature and management of mania in old age. Psychiatric Clinics of North America, 22, 649-65.

Starkstein S.E., Robinson R.G., Berthier, M.L., Parisk, R.M., y Price, T.R. (1988). Differential mood change following basal ganglia vs thalamic lesions. Archives of Neurology, 83, 725-30.

Strakowski, S.M., McElroy, S., Keckm, P., y West, S. (1994). The co-occurence of mania with medical and other psychiatric disorder. International Journal of Psychiatry in Medicine, 24, 305-28.

Ylikoski, R., Ylikoski, A., Erkinjuntti, T., Sulkava, R., Raininko, R., y Tilvis, R. (1993). White matter changes in healthy elderly persons correlate with attention and speed of mental processing. Archives of Neurology, 50, 818-24. 